\title{
Nonlinear Scale-Space Representation with Morphological Levelings
}

\author{
Fernand Meyer \\ Centre de Morphologie Mathématique, Ecole des Mines de Paris, 35, \\ Rue Saint Honoré, 77305 Fontainebleau, France \\ E-mail: meyer@cmm.ensmp.fr
}

and

Petros Maragos

Department of Electrical and Computer Engineering, National Technical University of Athens, Zografou 15773, Athens, Greece

E-mail: maragos@cs.ntua.gr

Received October 14, 1999, accepted November 14, 1999

\begin{abstract}
In this paper we present a nonlinear scale-space representation based on a general class of morphological strong filters, the levelings, which include the openings and closings by reconstruction. These filters are very useful for image simplification and segmentation. From one scale to the next, details vanish, but the contours of the remaining objects are preserved sharp and perfectly localized. Both the lattice algebraic and the scale-space properties of levelings are analyzed and illustrated. We also develop a nonlinear partial differential equation that models the generation of levelings as the limit of a controlled growth starting from an initial seed signal. Finally, we outline the use of levelings in improving the Gaussian scale-space by using the latter as an initial seed to generate multiscale levelings that have a superior preservation of image edges. () 2000 Academic Press
\end{abstract}

Key Words: scale-space; mathematical morphology; levelings; multiscale representation; differential equations.

\section{INTRODUCTION}

In many circumstances, the objects of interest which have to be detected, measured, segmented, or recognized in an image belong to a scale, and all remaining objects, to be discarded, to another scale. In some cases, however, such a threshold in the scales is not possible, and the information of interest is present at several scales; it has to be extracted from various scales. For such situations, multiscale approaches have been developed, where a series of coarser and coarser representations of the same image are derived. The recognition 
of the objects or segmentation will use the complete set of representations at various scales, not only the initial image.

A multiscale representation will be completely specified if one has defined the transformations from a finer scale to a coarser scale. To reduce the freedom of choice, some properties of these transformations may be specified. Invariance properties are the most general:

- Spatial invariance = invariance by translation.

- Isotropy = invariance by rotation.

- Invariance under illumination change: the transformation should commute with any anamorphosis, i.e., any pointwise increasing transformation, of the luminance.

One may add some requirements for the effect of the transformation itself:

- The transformation should really be a simplification of the image. As such it will not be reversible: some information has to be lost from one scale to the next.

- A particular form of simplification is expressed by the maximum principle: at any scale change, the maximum luminance at the coarser scale is always lower than the maximum luminance at the finer scale, the minimum always higher [6].

- Causality: coarser scales can only be caused by what happened at finer scales [8].

- The transformation should not create new structures at coarser scales; the most frequent requirement is that it should not create new regional extrema $[4,10]$.

Furthermore, if the goal is image segmentation, one may require that the contours remain sharp and not displaced. Finally, one has to care for the relations between the various scales. Many scale-space representations in the literature verify a semigroup property: if $f_{s}$ is the representation at scale $s$ of image $f$, then the representation at scale $t$ of $f_{s}$ should be the same as the representation at scale $s+t$ of $f: f_{s+t}=\left(f_{s}\right)_{t}$. In this paper we will present another structure by introducing an order relation among scales.

Since one rarely adds images, there is no particular reason, except mathematical tractability, to ask for linear transformations. If one chooses linearity, however, then various groups of the constraints listed above lead to the same solution: linear scale-space. The evolution of images with the scale follows the physics of luminance diffusion: the rate of change of luminance with scale is equal to the divergence of the luminance gradient [8]. The operator for changing scale is a convolution by a Gaussian kernel. Its major utility is to regularize the images, permitting to compute derivatives. Besides this advantage, linear scale-space cumulates the disadvantages. After convolution with a Gaussian kernel, the images are uniformly blurred, also the regions of particular interest like the edges. Furthermore, the localization of the structures of interest becomes extremely imprecise; if an object is found at one scale, one has to refine its contours along all finer scales. At very large scales, the objects are not recognizable at all, from excess blurring, but also from the appearance of spurious extrema in two dimensions. Various solutions have been proposed to reduce this problem. Some notable examples include Perona and Malik's anisotropic diffusion inhibited by high gradient values [21] and its improvement by Alvarez et al. and [2] using selective nonlinear image smoothing by mean curvature motion. Similar recent approaches include a tensor-dependent diffusion [30]. Such approaches reduce the problems but do not eliminate them completely: spurious extrema may still appear. 
Other nonlinear scale-spaces consider the evolution of curves and surfaces as a function of their geometry. Among them we find the morphological approaches. The basic ingredients of all multiscale morphological operators are the dilations and erosions of increasing size $[5,11,14]$. However, dilations and erosions by themselves cannot be used to represent the successive scales because they displace the image boundaries [7]. The first morphological scale-space approaches have been the granulometries associated to a continuous-scale family of openings or closings; openings operate only on the peaks and closings only on the valleys $[11,14]$. They obey a semigroup relation: $f_{\max (s, t)}=\left(f_{s}\right)_{t}$. The standard morphological openings (which are serial compositions of dilations and erosions) preserve well vertical image edges but may displace the horizontal contours; however, openings and closings do not create spurious extrema. A more powerful class of morphological filters that can also preserve the horizontal contours is the openings and closings by reconstruction $[23,29]$. These filters, starting from a reference signal $f$ consisting of several parts and a marker (initial seed) $g$ inside some of these parts, can reconstruct whole objects with exact preservation of their boundaries and edges. In this reconstruction process they simplify the original image by completely eliminating smaller objects inside which the marker cannot fit. Reconstruction filters have found numerous applications in a large variety of problems involving image enhancement and simplification, geometric feature detection, and segmentation. However, one of their disadvantages is that they treat asymmetrically the image foreground (peaks) and background (valleys). A symmetric treatment of peaks and valleys can be obtained using alternate sequential filters, which are extremely costly in terms of computation, especially if one uses openings and closings by reconstruction [25, 29]. A recent solution to this asymmetry problem came from the development of a more general powerful class of morphological filters, the levelings, introduced by Meyer [16, 17]. They have also been studied by Matheron [15] and Serra [26]. Levelings are transformations $\Lambda(f, g)$ that depend on two signals, the reference $f$ and the marker $g$, and include as special cases the reconstruction openings and closings.

In this paper, which is a union of our two previous works [13, 18], we present a new and extremely general nonlinear scale-space representation based on levelings with many extremely interesting features. The most interesting is the preservation of contours. Furthermore, no spurious extrema appear. As a matter of fact, the transformation from one scale to the next, called leveling, respects all the criteria listed above, except that it is not linear. From one scale to the next, the structures of the image progressively vanish, becoming flat or quasi-flat zones; however, as long they are visible, they keep exactly the same localization as in the initial image. In Section 2, we present an algebraic characterization and the scale-space properties of the simplest levelings. In Section 3 we show how to transform any marker function $g$ into a leveling of a function $f$ using discrete algorithms based on the algebraic definitions of levelings. We also present extensions of levelings and illustrate the algorithmic results with image examples. The scale-space analysis of levelings in Sections 2 and 3 is algebraic and based on lattice theory. A different formulation, based on ideas from dynamical systems and calculus, is presented in Section 4, where we develop a nonlinear partial differential equation (PDE) that can generate the leveling of a reference signal starting from a marker signal as initial condition. Finally we conclude in Section 5, where we also outline the use of levelings for improving the Gaussian scale-space by using the latter as an initial seed to generate multiscale levelings that have superior preservation of image edges and boundaries. 


\section{MULTISCALE REPRESENTATION THROUGH LEVELINGS}

\subsection{Flat and Quasi-flat Zones}

We are working here on gray-tone functions defined on a digital grid. We call the set of neighbors of a pixel $p N_{G}(p)$. The maximal (resp. minimal) value of a function $f$ within $N_{G}(p)$ represents the elementary dilation $\delta f$ (resp. erosion $\varepsilon f$ ) of the function $f$ at pixel $p$.

A path $P$ of cardinal $n$ between two pixels $p$ and $q$ on the grid $G$ is an $n$-tuple of pixels $\left(p_{1}, p_{2}, \ldots, p_{n}\right)$ such that $p_{1}=p$ and $p_{n}=q$, and for all $i,\left(p_{i}, p_{i+1}\right)$ are neighbors.

We will see that simple levelings are a subclass of connected operators [23], which means they extend flat zones and do not create new contours. More general levelings will extend quasi-flat zones, defined as follows.

Definition 2.1. Two pixels $x, y$ belong to the same R-flat zone of a function $f$ if and only if there exists an $n$-tuple of pixels $\left(p_{1}, p_{2}, \ldots, p_{n}\right)$ such that $p_{1}=x$ and $p_{n}=y$, and for all $i,\left(p_{i}, p_{i+1}\right)$ are neighbors and satisfy the symmetrical relation $f_{p_{i}} \mathrm{R} f_{p_{i}+1}$.

The simplest symmetrical relation $\mathrm{R}$ is equality, $f_{p_{i}}=f_{p_{i}+1}$, for which the quasi-flat zones are flat. As an example of a more complex relation $\mathrm{R}$, let us define for two neighboring pixels $p$ and $q, f_{p} \approx f_{q}$ by $\left|f_{p}-f_{q}\right| \leq \lambda$. This relation is symmetrical and defines quasi-flat zones with a maximal slope equal to $\lambda$.

\subsection{Characterization of Levelings}

We will define a nonlinear scale-space representation of images based on levelings. An image $g$ will be a representation of an image $f$ at a coarser scale if $g$ is a leveling of $f$, characterized by the following definition.

Definition 2.2. An image $g$ is a leveling of the image $f$ iff $\forall(p, q)$ neighbors: $g_{p}>g_{q} \Rightarrow f_{p} \geq g_{p}$ and $g_{q} \geq f_{q}$.

Remark. If the function $g$ is constant, no couple of neighboring pixels $(p, q)$ may be found for which $g_{p}>g_{q}$. Hence the implication $\left\{g_{p}>g_{q} \Rightarrow f_{p} \geq g_{p}\right.$ and $\left.g_{q} \geq f_{q}\right\}$ is always true, showing that a flat function is a leveling of any other function.

The relation $g$ is a leveling of $f$ will be written as $g \prec f$. The characterization using neighboring points, defining the levelings, is illustrated in Fig. 1b. In [17] we have shown that adopting a different order relation, giving a new meaning to $g_{p}>g_{q}$, leads to larger classes of levelings; such levelings create new quasi-flat zones and enlarge existing ones.

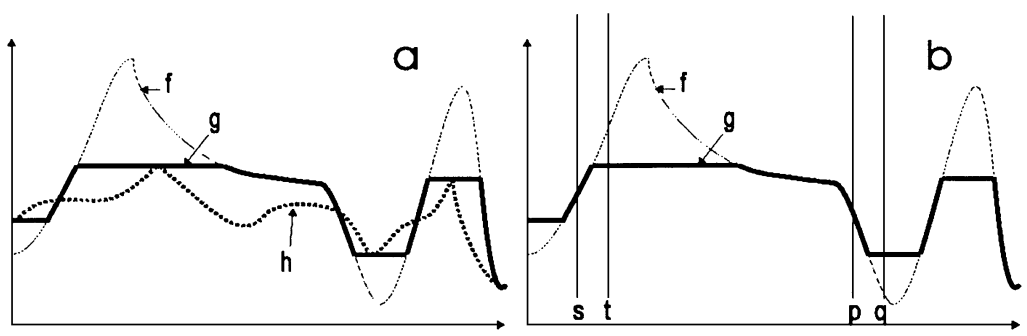

FIG. 1. (a) $f=$ reference function; $h=$ marker function; $g=$ associated leveling; (b) characterization of levelings on the transition zones. 
If the new order relation is written $g_{p} \succ g_{q}$ and its negation $g_{p} \preceq g_{q}$, then the symmetrical relation R of Definition 2.1 is defined by $\left\{g_{p} \mathrm{R} g_{q}\right\} \Leftrightarrow\left\{g_{p} \preceq g_{q}\right.$ and $\left.g_{q} \preceq g_{p}\right\}$.

\subsection{Properties of Levelings}

\subsubsection{Algebraic Properties}

If two functions $g_{1}$ and $g_{2}$ are both levelings of the same function $f$, then $g_{1} \vee g_{2}$ and $g_{1} \wedge g_{2}$ are both levelings of $f$. This property permits us to associate new levelings with a family of levelings. In particular if $\left(g_{i}\right)$ is a family of levelings of $f$, the morphological center $\left(f \vee \wedge g_{i}\right) \wedge \bigvee g_{i}$ of this family also is a leveling of $f$.

\subsubsection{Invariance Properties}

In the Introduction, we have listed a number of desirable properties of transformations on which to build a scale-space. They are obviously satisfied by levelings:

- Invariance by spatial translation.

- Isotropy: invariance by rotation.

- Invariance to a change of illumination: $g$ being a leveling of $f$, if $g$ and $f$ are submitted to the same increasing anamorphosis, then the transformed function $g^{\prime}$ will still be a leveling of the transformed function $f^{\prime}$.

\subsubsection{Relation between Two Scales}

Levelings will construct a scale-space when a true simplification of the image occurs between two scales. Let us now characterize the type of simplifications implied by levelings.

In this section we always suppose that $g$ is a leveling of $f$. As shown by the definition, if there is a transition for the function $g$ between two neighboring pixels $g_{p}>g_{q}$, then there exists an even greater transition between $f_{p}$ and $f_{q}$, as $f_{p} \geq g_{p}>g_{q} \geq f_{q}$. In other words, to any contour of the function $g$ corresponds a stronger contour of the function $f$ at the very same location, and the localization of this contour is exactly the same. This bracketing of each transition of the function $g$ by a transition of the function $f$ also shows that the "causality principle" is verified: coarser scales can only be caused by what happened at finer scales.

Furthermore, if we exclude the case where $g$ is a completely flat function, then the "maximum principle" is also satisfied: at any scale change, the maximal luminance at the coarser scale is always lower than the maximum intensity at the finer scale; the minimum is always larger.

Let us now analyze what happens in the zones where the leveling $g$ departs from the function $f$. Let us consider two neighboring points $(p, q)$ for which $f_{p}>g_{p}$ and $f_{q}>g_{q}$. For such a couple of pixels, the second half $\left(f_{p} \geq g_{p}\right.$ and $\left.g_{q} \geq f_{q}\right)$ of the implication-defining leveling is wrong, showing that the first half must also be wrong; i.e., $g_{p} \leq g_{q}$. By reason of symmetry we also have $g_{p} \geq g_{q}$, and hence $g_{p}=g_{q}$. This means that if $g$ is a leveling of $f$, the connected components of the antiextensivity zones $\{f>g\}$ are necessarily flat. By duality, the same holds for the extensivity zones $\{f<g\}$.

The last criterion, "no new extrema at larger scales," also is satisfied, as shown by the following section. 


\subsubsection{Life and Death of the Regional Minima and Maxima}

Levelings are a particular case of monotone planings:

DEFINITION 2.3. An image $g$ is a monotone planing of the image $f$ iff $\forall(p, q)$ neighbors: $g_{p}>g_{q} \Rightarrow f_{p}>f_{q}$.

THEOREM 2.1. A monotone planing does not create regional minima or maxima. In other words, if $g$ is a monotone planing of $f$, and if $g$ has a regional minimum (resp. maximum) $X$, then $f$ possesses a regional minimum (resp. maximum) $Z \subset X$.

Hint of the Proof. If $X$ is a regional minimum of $g$, all its neighbors have a higher altitude. To these increasing transitions of $g$ correspond increasing transitions of $f$. It is then easy to show that the lowest pixel for $f$ within $X$ belongs to a regional minimum $Z$ for $f$ included in $X$.

\subsubsection{Relations between Multiple Scales: Preorder Relation}

We have now to consider the relations between multiple scales. Until now, we have presented how levelings simplify images. To speak about scales, we need some structure among scales. This structure is a lattice structure. To be a leveling is in fact an order relation as shown by the following two lemmas.

Lemma. The relation $\{g$ is a leveling of $f\}$ is symmetric and transitive: it is a preorder relation.

LEMma. The family of levelings, from which we exclude the trivial constant functions, verify the anti-symmetry relation: if $f$ is a nonconstant function and a leveling of $g$, and simultaneously $g$ is a leveling of $f$, then $f=g$.

Being an anti-symmetric preorder relation, the relation $\{g$ is a leveling of $f\}$ is an order relation, except for functions which are constant everywhere. With the help of this order relation, we are now able to construct a multiscale representation of an image in the form of a series of levelings $\left(g_{0}=f, g_{1}, \ldots, g_{n}\right)$, where $g_{k}$ is a leveling of $g_{k-1}$, and as a consequence of the transitivity, $g_{k}$ is also a leveling of each function $g_{j}$ for $j<k$.

\section{CONSTRUCTION OF THE LEVELINGS}

\subsection{A Criterion for $\mathrm{C}$ haracterizing Levelings}

It will be fruitful to consider the levelings as the intersection of two larger classes, the lower levelings and the upper levelings, defined as follows.

Definition 3.1. A function $g$ is a lower leveling of a function $f$ if and only if for any couple of neighboring pixels $(p, q): g_{p}>g_{q} \Rightarrow g_{q} \geq f_{q}$.

Definition 3.2. A function $g$ is an upper leveling of a function $f$ if and only if for any couple of neighboring pixels $(p, q): g_{p}>g_{q} \Rightarrow g_{p} \leq f_{p}$.

The name "upper leveling" comes from the fact that all connected components where $g>f$ are flat: for any couple of neighboring pixels $(p, q)$ :

$$
\left|\begin{array}{l}
g_{q}>f_{q} \\
g_{p}>f_{p}
\end{array}\right| \Rightarrow g_{p}=g_{q} .
$$


Similarly if $g$ is a lower leveling of $f$, then all connected components where $g<f$ are flat.

Obviously, a function $g$ is a leveling of a function $f$ if and only if it is both an upper and a lower leveling of the function $f$. Let us now propose an equivalent formulation for the lower levelings:

Criterion. A function $g$ is a lower leveling of a function $f$ if and only if for each pixel $q$ with a neighbor $p$ verifying $g_{p}>g_{q}$ the relation $g_{q} \geq f_{q}$ is satisfied.

But the pixels with this property are those for which the dilation $\delta$ will increase the value; i.e., $g_{q}<\delta_{q} g$. This leads to a new criterion:

Criterion. A function $g$ is a lower leveling of a function $f$ if and only if $g_{q}<\delta_{q} g \Rightarrow$ $g_{q} \geq f_{q}$.

Recalling that the logical meaning of $[A \Rightarrow B]$ is [not $A$ or $B]$ we may interpret $\left[g_{q}<\right.$ $\left.\delta_{q} g \Rightarrow g_{q} \geq f_{q}\right]$ as $\left[g_{q} \geq \delta_{q} g\right.$ or $\left.g_{q} \geq f_{q}\right]$ or in a equivalent manner $\left[g_{q} \geq f_{q} \wedge \delta_{q} g\right]$. This gives the following criterion.

Criterion Low. A function $g$ is a lower leveling of a function $f$ if and only if $g \geq f \wedge \delta g$.

In a similar way we derive a criterion for upper levelings:

Criterion Up. A function $g$ is an upper leveling of a function $f$ if and only if $g \leq f \vee \varepsilon g$.

Putting everything together yields a criterion for levelings.

Criterion. A function $g$ is a leveling of a function $f$ if and only if $f \wedge \delta g \leq g \leq f \vee \varepsilon g$ (see [15]).

\subsection{Openings and Closings by Reconstruction}

We recall that a function $g$ is an opening (resp. closing) by reconstruction of a function $f$ iff $g=f \wedge \delta g$ (resp. $g=f \vee \varepsilon g$ ). As it verifies the criterion Low (resp. Up), such a function $g$ is then a lower (resp. upper) leveling of $f$. The reciprocal is also true. Hence:

PROPOSITION 3.1. $g$ is an opening (resp. closing) by reconstruction of a function $f$ if and only if $g$ is a lower (resp. upper) leveling of $f$ verifying $g \leq f$ (resp. $g \geq f$ ).

Using this characterization, we may particularize the initial definition of lower levelings in the case where $f \geq g$ :

PROPOSITION 3.2. $g$ is an opening by reconstruction of a function $f$ if and only if $g \leq f$ and for any couple of neighboring pixels $(p, q): g_{p}>g_{q} \Rightarrow g_{q}=f_{q}$.

PROPOSITION 3.3. $g$ is a closing by reconstruction of a function $f$ if and only if $g \geq f$ and for any couple of neighboring pixels $(p, q): g_{p}>g_{q} \Rightarrow g_{p}=f_{p}$.

Remark. If $g$ is a leveling or lower leveling of $f$, then $g \wedge f$ is a lower leveling of $f$ verifying $g \wedge f \leq f$, i.e., an opening by reconstruction of $f$. Similarly if $g$ is a leveling or upper leveling of $f$, then $g \vee f$ is a closing by reconstruction of $f$.

\subsection{An Algorithm for C onstructing L evelings}

We finally adopt the following general criterion for levelings. 
Criterion. A function $g$ is a leveling of a function $f$ if and only if $f \wedge \alpha g \leq g \leq f \vee \beta g$, where $\alpha$ is an extensive operator; i.e., $\alpha g \geq g$, and $\beta$ is an antiextensive operator; i.e., $\beta g \leq g$.

With the help of this criterion, we may turn each function $g$ into the leveling of a function $f$. We will call the function $f$ the reference function and the function $g$ the marker function. Given two functions $g$ and $f$, we want to transform $g$ into a leveling of $f$. If $g$ is not a leveling of $f$, then the criterion $[f \wedge \alpha g \leq g \leq f \vee \beta g]$ is false for at least a pixel $p$. The criterion is not verified in two cases:

- $g_{p}<f_{p} \wedge \alpha_{p} g$. Hence the smallest modification of $g_{p}$ for which the criterion becomes true is $g_{p}^{\prime}=f_{p} \wedge \alpha_{p} g$. We remark that $g_{p} \leq g_{p}^{\prime} \leq f_{p}$.

- $g_{p}>f_{p} \vee \beta_{p} g$. Hence the smallest modification of $g_{p}$ for which the criterion becomes true is $g_{p}^{\prime}=f_{p} \vee \beta_{p} g$. We remark that $g_{p} \geq g_{p}^{\prime} \geq f_{p}$.

We remark that for $\left\{g_{p}=f_{p}\right\}$ the criterion is always satisfied. Hence another formulation of the algorithm:

- $l e v^{-}:$On $\{g<f\}$ do $g=f \wedge \alpha g$.

- $l e v^{+}:$On $\{g>f\}$ do $g=f \vee \beta g$.

It is easy to check that this algorithm amounts to replacing $g$ by the new value $g=(f \wedge$ $\alpha g) \vee \beta g=(f \vee \beta g) \wedge \alpha g$ everywhere.

We repeat the algorithm until the criterion is satisfied everywhere. We are certain that the algorithm will converge, since the modifications of $g$ are pointwise monotonics: the successive values of $g$ get closer and closer to $f$ until convergence.

To optimize the speed of the algorithm, we use a unique parallel step of the algorithm, $g=(f \wedge \alpha g) \vee \beta g$. After this first step the algorithms $\left[l e v^{-}\right]$and $\left[l e v^{+}\right]$have no effect on each other and may be used in any order. In particular one may use them as sequential algorithms in which the new value of any pixel is used to compute the values of the neighboring pixels. This may be done during alternating raster scans, a direct scan from top to bottom and left to right being followed by an inverse scan from bottom to top and right to left. Or hierarchical queues may be used, allowing us to process the pixels in decreasing order on $\{g<f\}$ and in increasing order on $\{g>f\}$.

Let us illustrate in Fig. 1a how a marker function $h$ is transformed until it becomes a function $g$ which is a leveling of $f$. This leveling uses for $\alpha$ the dilation $\delta$ and for $\beta$ the erosion $\varepsilon$. On $\{h<f\}$, the leveling increases $h$ as little as possible until a flat zone is created or the function $g$ hits the function $f$; hence on $\{g<f\}$, the function $g$ is flat. On $\{h>f\}$, the leveling decreases $h$ as little as possible until a flat zone is created or the function $g$ hits the function $f$; hence on $\{g>f\}$, the function $g$ also is flat. For more general levelings, quasi-flat zones are created.

If $g$ is not modified, while this complete algorithm is applied to a couple of functions $(f, g)$, then $g$ is a leveling of $f$. If, on the other hand, $g$ is modified, one repeats the same algorithm until convergence as explained above.

\subsection{R obustness of Levelings}

In this section, we will see that levelings are particularly robust: they are strong morphological filters. We recall that an operator $\psi$ is called a morphological filter if it is:

- Increasing: $g>h \Rightarrow \psi g>\psi h$. This implies that $\psi(h \wedge k)<\psi h \wedge \psi k$ and $\psi(h \vee$ $k)>\psi h \vee \psi k$. 
- Idempotent: $\psi \psi=\psi$. This means that the operator is stable: it is sufficient to apply it once in order to get the final result. (For instance, the median filter, which is not a morphological filter, is not stable and may oscillate when iterated.)

A morphological filter is called strong, if furthermore $\psi(i d \vee \psi)=\psi(i d \wedge \psi)=\psi$, where $i d$ represents the identity operator. This property defines that functions within a given range will yield the same result; i.e., for any function $h$ satisfying $f \wedge \psi f<h<f \vee \psi f$, we have $\psi f=\psi h$.

In our case, we have the leveling $\Lambda(f, g)$, which we view as an operator mapping an input signal $f$ to its leveling. For a fixed marker function $g$ and a varying reference function $f$, this operator is a strong morphological filter. If we call the opening by reconstruction of $f$ based on the marker $g \Lambda^{-}(f, g)$ and the closing by reconstruction $\Lambda^{+}(f, g)$, it can be shown that

$$
\Lambda(f, g)=\Lambda^{-}\left(\Lambda^{+}(f, g), g\right)=\Lambda^{+}\left(\Lambda^{-}(f, g), g\right)
$$

Thus, the leveling is a commutative product of a reconstruction opening followed by a reconstruction closing, which is a sufficient condition for a leveling to be a strong morphological filter.

Levelings depend upon several parameters. First, the type of leveling has to be chosen, which depends upon the choice of the operators $\alpha$ and $\beta$. Figure 2 presents three different levelings, applied to the same reference and marker image. The operators $\alpha$ and $\beta$ used for producing them are, from left to right, the following: (1) $\alpha=\delta, \beta=\varepsilon$; (2) $\alpha=i \mathrm{~d} \vee$ $(\delta-1), \beta=\mathrm{id} \wedge(\varepsilon+1)$; (3) $\alpha=\mathrm{id} \vee \gamma \delta, \beta=\mathrm{id} \wedge \varphi \varepsilon$, where $\gamma$ and $\varphi$ are respectively an opening and a closing. In Fig. 3a flat leveling based on $\delta$ and $\varepsilon$ is applied to the same reference image (in the center of the figure), using different markers produced by an alternate sequential filter applied to the reference image, marker 1 using disks as structuring elements and marker 2 using line segments.

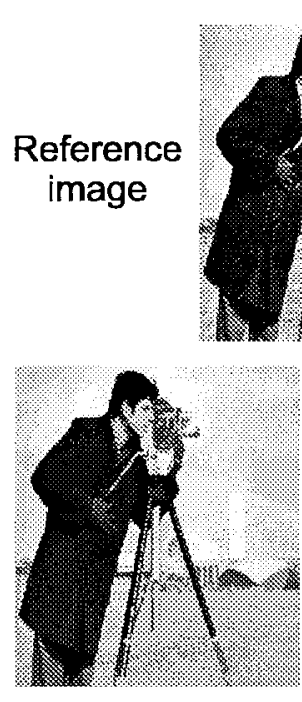

leveling 1

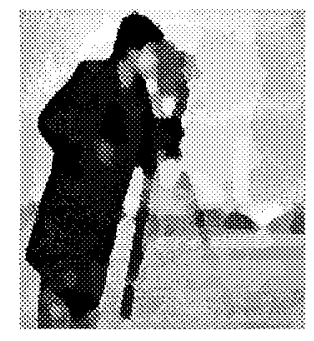

Marker image

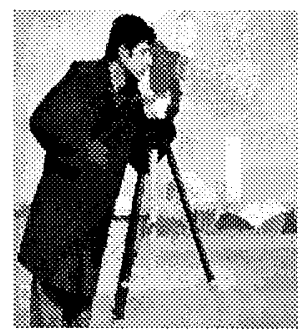

leveling 3

FIG. 2. Three different levelings applied to the same reference and marker image. 


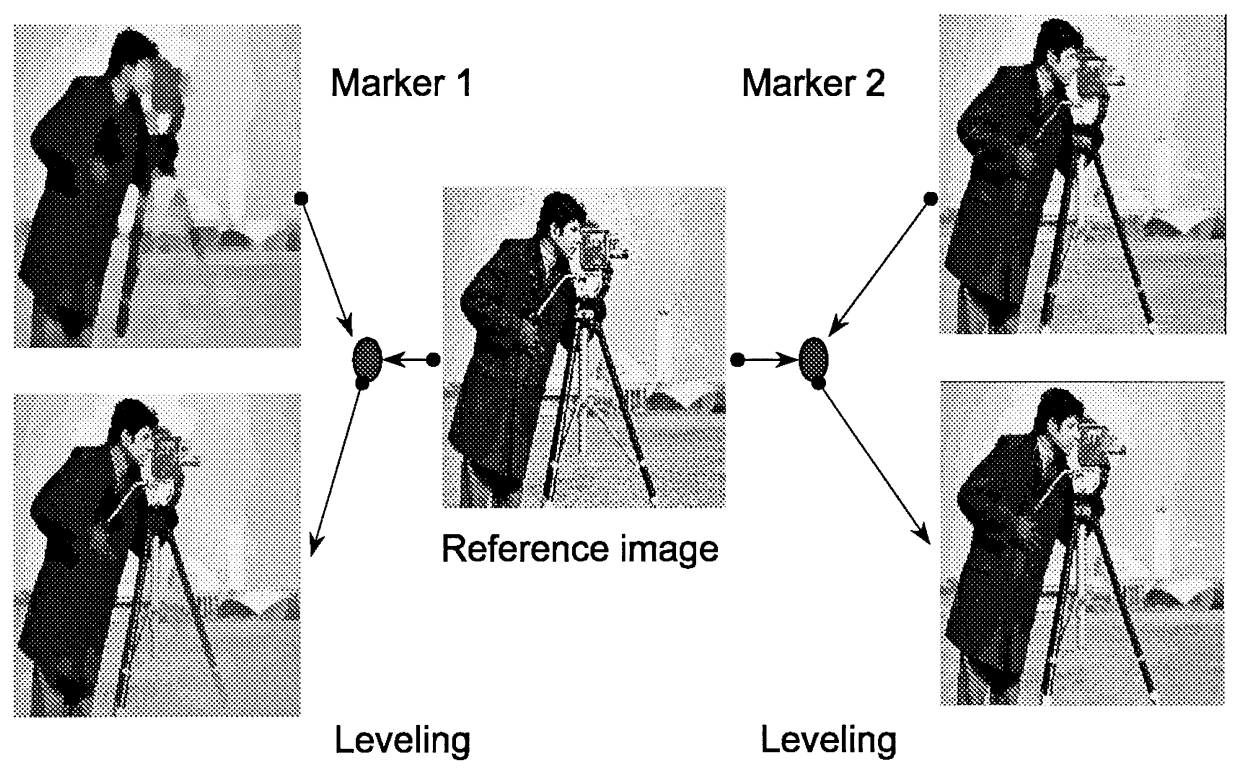

FIG. 3. A leveling applied to the same reference image with distinct marker images.

\subsection{Multiscale Levelings}

We have now to consider the multiscale aspect, that is, when more than one scale is under consideration. Levelings permit us to construct various multiscale families. Let us present a few of them.

\subsubsection{Levelings Associated to Monotone Families of Erosions and Dilations}

We use here a family of leveling operators based on a family $\left(\alpha_{i}\right)$ of extensive dilations and the corresponding family of adjunct erosions $\left(\beta_{i}\right)$, satisfying $\alpha_{i}<\alpha_{j}$ and $\beta_{i}>\beta_{j}$ for $i>j$. We call $\Lambda^{i} f$ the leveling of $f$ built with $\alpha_{i}$ and $\beta_{i}$ and with a fixed marker $g$. That is, $\Lambda^{i} f$ is the leveling obtained by iterating until convergence the operator $g=\left(f \wedge \alpha_{i} g\right) \vee$ $\beta_{i} g=\left(f \vee \beta_{i} g\right) \wedge \alpha_{i} g$.

It is easy to verify that for $i>j$ we have $f \wedge \Lambda^{i} f \leq \Lambda^{j} f \leq f \vee \Lambda^{i} f$. But since $\Lambda^{i}$ a strong operator, these inequalities imply that $\Lambda^{i} \Lambda^{j} f=\Lambda^{i} f$. This means that $\Lambda^{i} f$ also is a leveling of each $\Lambda^{j} f$ for $i>j$.

As an example one may construct slope levelings with increasing slopes by setting $\alpha_{i}=\mathrm{id} \vee(\delta-i), \beta_{i}=\mathrm{id} \wedge(\varepsilon+i)$.

\subsubsection{Levelings Associated to an Arbitrary Family of Marker Functions}

Consider a fixed leveling $\Lambda$ associated to an extensive dilation $\alpha$ and the adjunct erosion $\beta$. We may use an arbitrary family of marker functions $g_{i}, i=1,2,3, \ldots$, and consider the associated levelings: we write $\Lambda_{g_{i}} f$ for the leveling of $f$ associated to the marker $g_{i}$. We may then construct an increasing family of levelings associated with the family of markers by the following mechanism:

$$
\ell_{1}=\Lambda_{g_{i}} f, \ell_{2}=\Lambda_{g_{2}} \Lambda_{g_{1}} f, \ldots, \ell_{n}=\Lambda_{g_{n}} \Lambda_{g_{n-1}} \cdots \Lambda_{g_{2}} \Lambda_{g_{1}} f
$$

The above sequence of steps ensures that $\ell_{j}$ is a leveling of $\ell_{i}$ for $j>i$. This is due to the fact that "to be a leveling" is a transitive relation. Based on this idea, Fig. 4 presents one 

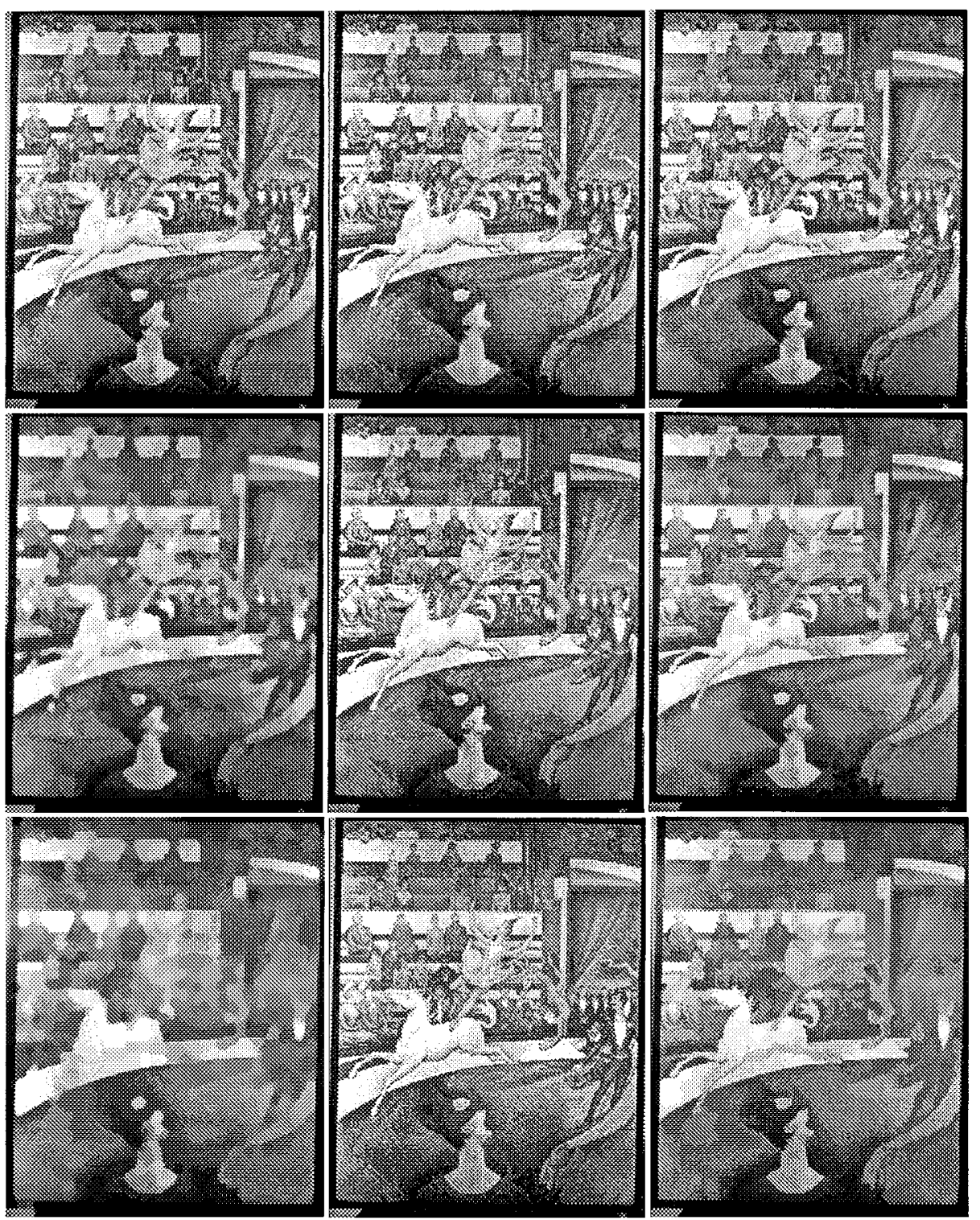

FIG. 4. Illustration of a multiscale leveling representation with markers produced by alternate sequential filtering.

example of how levelings can be used to derive a multiscale representation of an image. In Fig. 4 the multiscale markers are produced by alternate sequential filters with disks; i.e., $g_{0}=f$ is the original image and $g_{i}=\varphi_{i} \gamma_{i} g_{i-1}$ for $i=1,2,3$, where $\varphi_{i}$ and $\gamma_{i}$ are standard closings and openings by a disk of radius $i$. The levelings are produced by following the sequential hierarchy of (2). The markers $\left(g_{1}, g_{3}, g_{5}\right)$ and the leveling images $\left(\ell_{1}, \ell_{3}, \ell_{5}\right)$ shown in Fig. 4 are arranged as follows: 
Semigroup property of flat levelings. In the case where the leveling $\Lambda$ is the flat leveling associated to the dilation $\delta$ and the adjunct erosion $\varepsilon$, we have a stronger property, in the form of a semigroup. Let us denote by $\Lambda_{g} f$ the leveling of $f$ associated with the marker $g$.

Then for two different markers $g$ and $k$ we have the absorption property: $\Lambda_{g} \Lambda_{k} \Lambda_{g} f=$ $\Lambda_{k} \Lambda_{g} f$ and $\Lambda_{g} \Lambda_{g} \Lambda_{k} f=\Lambda_{g} \Lambda_{k} f$. It follows that the operator defined as

$$
L_{n}=\Lambda_{g_{n}} \Lambda_{g_{n-1}} \cdots \Lambda_{g_{2}} \Lambda_{g_{1}}
$$

satisfies the following semigroup property:

$$
L_{n} L_{m}=L_{m} L_{n}=L_{\max (m, n)}
$$

This implies in particular that $L_{n}$ is idempotent $(n=m)$. Since it is also increasing, it is a morphological filter.

In $B_{n}$ is the invariance set of $L_{n}$, i.e., $B_{n}=\left\{f \mid L_{n} f=f\right\}$, the preceding semigroup property implies that $B_{n}=L_{n}\left(B_{m}\right)$ and $B_{n} \subset B_{m}$ for $n>m$.

\subsubsection{Levelings Associated to an Alternating Family of Marker Functions}

Consider again the fixed leveling $\Lambda$ associated to an extensive dilation $\alpha$ and the adjunct erosion $\beta$. But now the family of marker functions $g_{i}, i=1,2,3, \ldots$, is an alternating family, because they satisfy $g_{2 k} \leq \cdots \leq g_{4} \leq g_{2} \leq f \leq g_{1} \leq g_{3} \leq \cdots \leq g_{2 k-1}$. Using the same notation as above, it is easy to verify that $\Lambda_{g_{i}}$ is a reconstruction opening for any even index $i$ and a reconstruction closing for any odd index $i$. Hence $L_{n}=\Lambda_{g_{2 n}} \Lambda_{g_{2 n-1}} \cdots \Lambda_{g_{2}} \Lambda_{g_{1}} f$ is an alternating sequential filter, which obeys the usual semigroup property of such filters [25]: $L_{j} L_{i}=L_{j}$ for $j>i$, but $L_{i} L_{j} \leq L_{j}$.

\section{A PDE GENERATING LEVELINGS}

In computer vision continuous models for scale-space image analysis based on partial differential equations have been proposed. Motivations for using PDEs include better and more inuitive mathematical modeling, connections with physics, and better approximation to the Euclidean geometry of the problem. Inspired by the use of the classic heat PDE to model the linear (Gaussian) scale-space [8], in 1992 three teams of researchers (Alvarez et al. [1], Brockett and Maragos [5], and Van den Boomgaard and Smeulders [28]) independently published nonlinear PDEs that model the nonlinear scale-space of elementary morphological operators; each team focused on different aspects of the problem. The PDEs for flat dilations and erosions by disks were numerically implemented by Arehart et al. [3] and Sapiro et al. [24] using the Osher and Sethian [20] algorithm for solving Hamilton-Jacobi PDEs of the curve evolution type. These implementations demonstrated the superiority of the performance of the PDE approach over that of discrete morphology in terms of isotropy and subpixel accuracy. A unified view of this new approach to mathematical morphology and related problems based on differential equations and dynamical systems was presented by Maragos [12].

In the rest of this section we shall present a PDE for levelings, first introduced in [13]. The two basic ingredients in developing such a PDE are the PDEs for generating dilations and 
erosions. Therefore, before presenting the new PDE for levelings we review some PDEs ${ }^{1}$ required for multiscale morphology.

\subsection{PDE s for D ilations and E rosions}

All multiscale morphological operations, at their most basic level, are generated by multiscale dilations and erosions, which are obtained by replacing in the standard translationinvariant dilations and erosions the unit-scale kernel (structuring element) $k(x, y)$ with a multiscale version $k^{(t)}(x, y) \triangleq t k(x / t, y / t), t>0$. The multiscale dilation of a 2D signal $f(x, y)$ by $k^{(t)}$ is the space-scale function

$$
\delta(x, y, t) \triangleq\left(f \oplus k^{(t)}\right)(x, y)=\sup _{(a, b)}\{f(x-a, y-b)+t k(a / t, b / t)\}, \quad t>0,
$$

where $\delta(x, y, 0)=f(x, y)$. Similarly, the multiscale erosion of $f$ is defined as

$$
\varepsilon(x, y, t) \triangleq\left(f \ominus k^{(t)}\right)(x, y)=\inf _{(a, b)}\{f(x+a, y+b)-t k(a / t, b / t)\} .
$$

For 2D signals $f(x, y)$, and if $k(x, y)$ is the $0 /-\infty$ indicator function of the unit disk, then the PDEs generating the multiscale flat dilation $\delta(x, y, t)$ and erosion $\varepsilon(x, y, t)$ of $f$ are

$$
\delta_{t}=\|\nabla \delta\|=\sqrt{\left(\delta_{x}\right)^{2}+\left(\delta_{y}\right)^{2}}, \quad \varepsilon_{t}=-\|\nabla \varepsilon\|,
$$

with initial values $\delta(x, y, 0)=\varepsilon(x, y, 0)=f(x, y)$.

These simple but nonlinear PDEs are satisfied at points where the data are smooth, i.e., where the partial derivatives exist. However, even if the initial image/signal $f$ is smooth, at finite scales $t>0$ the above multiscale dilation evolution may create discontinuities in the derivatives of $\delta$, called shocks, which then continue propagating in scale-space. Thus, the multiscale dilations are weak solutions of the corresponding PDEs.

The above PDEs for dilations of gray-level images by flat structuring elements directly apply to binary images, because flat dilations commute with thresholding and hence, when the gray-level image is dilated, each one of its thresholded versions representing a binary image is simultaneously dilated by the same element and at the same scale. However, this is not the case with gray-level structuring functions. For example, if $k(x, y)=$ $-a\left(x^{2}+y^{2}\right), a>0$, is an infinite-support parabolic function, the dilation PDE becomes

$$
\delta_{t}=\left[\left(\delta_{x}\right)^{2}+\left(\delta_{y}\right)^{2}\right] / 4 a .
$$

\subsection{PDE for Levelings}

Consider a $2 \mathrm{D}$ signal $f(x, y)$ and a marker signal $g(x, y)$ from which a leveling $\Lambda(f, g)$ will be produced.

If $g \leq f$ everywhere and we start iteratively growing $g$ via incremental flat dilations with a disk of an infinitesimally small radius $\Delta t$ but without ever growing the result above the graph of $f$, then in the limit we shall have produced the opening by reconstruction of $f$ (with respect to the marker $g$ ), which is a special leveling. The infinitesimal generator of this

\footnotetext{
${ }^{1}$ Notation for PDEs: For $u=u(x, y, t), u_{t}=\partial u / \partial t, u_{x}=\partial u / \partial x, u_{y}=\partial u / \partial y, \nabla u=\left(u_{x}, u_{y}\right)$.
} 
signal evolution can be modeled via a dilation PDE that has a mechanism to stop the growth whenever the intermediate result attempts to create a function larger than $f$. Specifically, let $u(x, y, t)$ represent the evolutions of $f$ with initial value $u_{0}(x, y)=u(x, y, 0)=g(x, y)$. Then $u$ is a weak solution of the initial-value PDE system

$$
\begin{aligned}
\frac{\partial u}{\partial t} & =\operatorname{sign}(f-u) \sqrt{\left(\frac{\partial u}{\partial x}\right)^{2}+\left(\frac{\partial u}{\partial y}\right)^{2}} \\
u(x, y, 0) & =g(x, y),
\end{aligned}
$$

where $\operatorname{sign}(r)$ is equal to +1 if $r>0,-1$ if $r<0$, and 0 if $r=0$. Since $g \leq f$, the above initialvalue PDE system models a conditional dilation that grows the intermediate result as long as it does not exceed $f$. In the limit we obtain the final result $u_{\infty}(x, y)=\lim _{t \rightarrow \infty} u(x, y, t)$. The mapping $u_{0} \mapsto u_{\infty}$ is the opening by reconstruction filter.

If in the above paradigm we reverse the order between $f$ and $g$, i.e., assume that $g \geq f$, and replace the positive growth (dilation) of $g$ with negative growth via erosion that stops when the intermediate result attempts to become smaller than $f$, then we obtain the closing by reconstruction of $f$ with respect to the marker $g$. This is another special case of a leveling whose generation can also be modeled by the same PDE (6) but with a marker that exceeds $f$. This dynamical system models a conditional erosion that keeps reducing the intermediate result as long as it does not decrease below $f$.

What happens if we use any of the above PDEs when there is no specific order between $f$ and $g$ ? In such a case the PDE (6) has a varying coefficient sign $(f-u)$ with spatiotemporal dependence which controls the instantaneous growth and stops it whenever $f=u$. (Of course, there is no growth also at stationary points where $\nabla u=0$.) The control mechanism is of a switching type: For each $t$, at pixels $(x, y)$ where $u(x, y, t)<f(x, y)$ it acts as a dilation PDE and hence shifts outward the surface of $u(x, y, t)$ but does not move the extrema points. Wherever $u(x, y, t)>f(x, y)$ the PDE acts as an erosion PDE and reverses the direction of propagation. The final result $u_{\infty}(x)=\lim _{t \rightarrow \infty} u(x, t)$ is a general leveling of $f$ with respect to $g$. We call (6) a switched dilation PDE. The switching action of this PDE model occurs at zero crossings of $f-u$ where shocks are developed. Obviously, the PDEs generating the opening and closing by reconstruction are special cases where $g \leq f$ and $g \geq f$, respectively. However, the PDEs generating the reconstruction filters do not involve switching of growth.

The switching between dilation- and erosion-type PDEs also occurs in a class of nonlinear time-dependent PDEs which was proposed in [19] to deblur images and/or enhance their contrast by generating shocks and hence sharpening edges. For 2D images a special case of such a PDE is

$$
u_{t}=-\|\nabla u\| \operatorname{sign}\left(\nabla^{2} u\right)
$$

A major conceptual difference between the above edge-sharpening PDE and our PDE generating levelings is that in the former the switching is determined by the edges, i.e., the zero crossing points of the Laplacian of $u$, whereas in the latter the switching is controlled by comparing $u$ against the external reference signal $f$. Note also that, if at some point there is an edge in the leveling output, then there must exist an edge of equal or bigger size in the initial (reference) image. 


\subsection{D iscretization Algorithm and Experiments}

To produce a shock-capturing and entropy-satisfying numerical method for solving the general leveling PDE (6), we use ideas from the technology of solving PDEs corresponding to hyperbolic conservation laws [9] and Hamilton-Jacobi formulations [20]. Thus, we propose the following discretization sheme, which is an adaptation of a scheme proposed in [19] for solving (7).

Let $U_{i, j}^{n}$ be the approximation of $u(x, y, t)$ on a grid $\left.(i \Delta x, j \Delta y, n \Delta t)\right)$. Consider the spatial forward and backward difference operators:

$$
D_{+x} U_{i, j}^{n} \triangleq \frac{U_{i+1, j}^{n}-U_{i, j}^{n}}{\Delta x}, \quad D_{-x} U_{i, j}^{n} \triangleq \frac{U_{i, j}^{n}-U_{i-1, j}^{n}}{\Delta x} .
$$

Similarly we define the difference operators $D_{+y}$ and $D_{-y}$ along the $j$ direction. Then we approximate the leveling PDE (6) by the nonlinear difference equation

$$
\begin{aligned}
& U_{i, j}^{n+1}=U_{i, j}^{n} \\
& -\Delta t\left[\cdots\left(S_{i, j}^{n}\right)^{+} \sqrt{\left(\left(D_{-x} U_{i, j}^{n}\right)^{+}\right)^{2}+\left(\left(D_{+x} U_{i, j}^{n}\right)^{-}\right)^{2}+\left(\left(D_{-y} U_{i, j}^{n}\right)^{+}\right)^{2}+\left(\left(D_{+y} U_{i, j}^{n}\right)^{-}\right)^{2}}\right. \\
& \left.+\left(S_{i, j}^{n}\right)^{-} \sqrt{\left(\left(D_{+x} U_{i, j}^{n}\right)^{+}\right)^{2}+\left(\left(D_{-x} U_{i, j}^{n}\right)^{-}\right)^{2}+\left(\left(D_{+y} U_{i, j}^{n}\right)^{+}\right)^{2}+\left(\left(D_{-y} U_{i, j}^{n}\right)^{-}\right)^{2}}\right],
\end{aligned}
$$

where $S_{i, j}^{n}=\operatorname{sign}\left(f(i \Delta x, j \Delta y)-U_{i, j}^{n}\right)$, and we denote $(r)^{+}=\max (r, 0),(r)^{-}=\min (r, 0)$ for any real $r$. For stability, $(\Delta t / \Delta x+\Delta t / \Delta y) \leq 0.5$ is required. Further, at each iteration we enforce the sign consistency

$$
\operatorname{sign}\left(U^{n}-f\right)=\operatorname{sign}(g-f) .
$$

We have not proved theoretically that the above iterated scheme converges when $n \rightarrow \infty$, but through many experiments we have observed that it converges in a finite number of steps. Three examples of the action of the above 2D algorithm are shown in Fig. 5.

\subsection{PDE s for Levelings with Quasi-F lat Zones}

The levelings produced by running the PDE (6) consist of portions of the original reference signal and of flat zones. Actually they enlarge the flat zones of the reference signal. Is it possible to generate via PDEs generalized levelings that have quasi-flat zones (for example, zones with constant linear slope or zones with parabolic surface)? The answer is yes. We explain it via the parabolic example. If we replace the flat dilation PDE generator in (6) with the PDE generator for multiscale dilations by a $2 \mathrm{D}$ unit-scale parabola $k(x, y)=$ $-a\left(x^{2}+y^{2}\right)$ we obtain the PDE for 2D parabolic levelings:

$$
\begin{aligned}
u_{t} & =\frac{1}{4 a}\|\nabla u\|^{2} \operatorname{sign}(f-u) \\
u(x, y, 0) & =g(x, y) .
\end{aligned}
$$




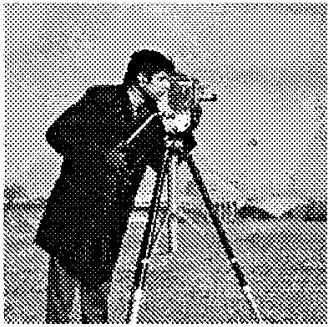

(a)

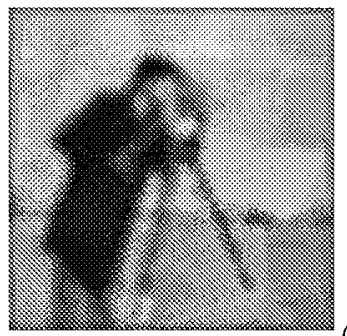

(b)
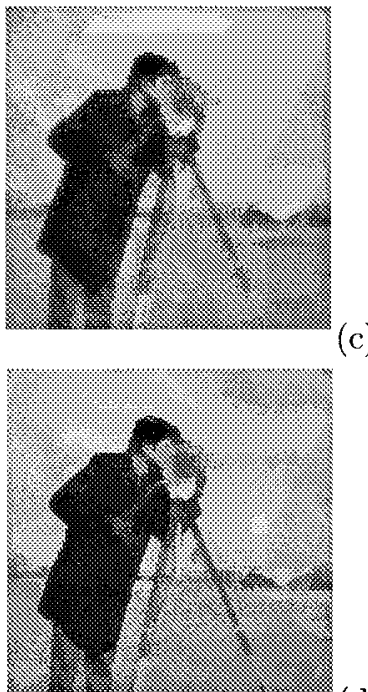

(d)

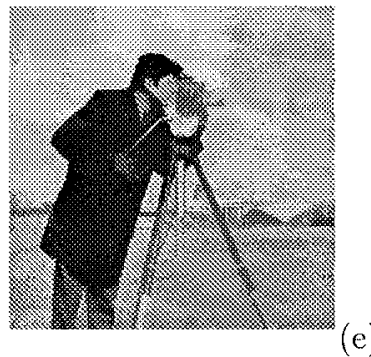

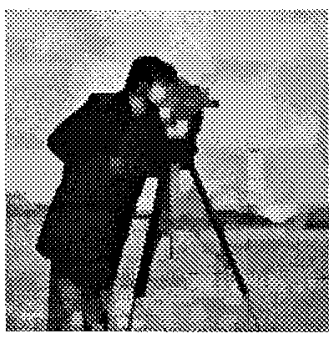

(f)

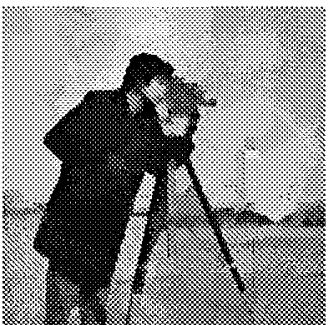

(g)

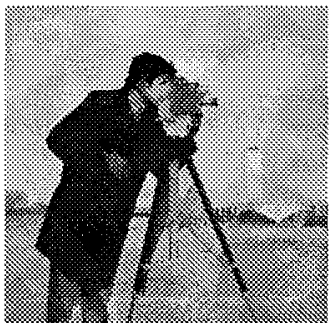

(h)

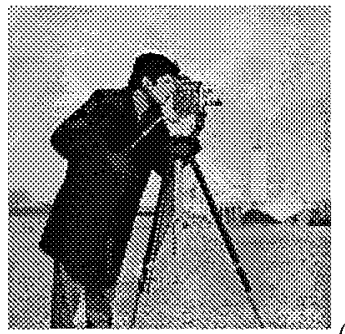

(i)

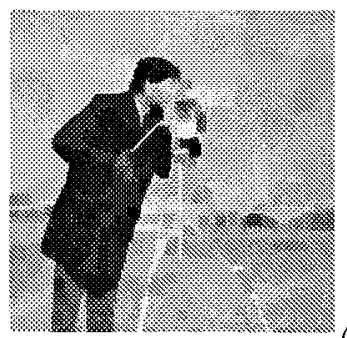

(j)

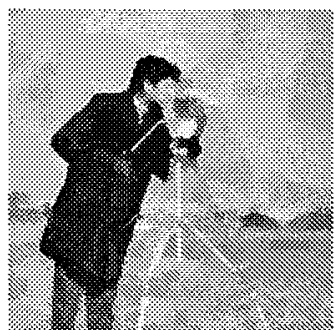

(k)

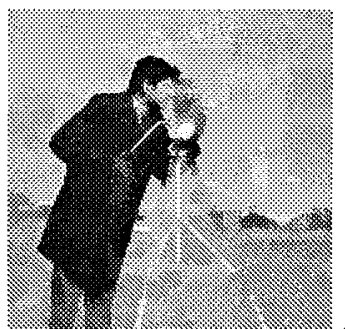

(1)

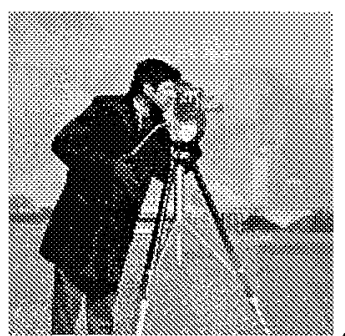

$(\mathrm{m})$

FIG. 5. Evolutions of the 2D leveling PDE on the reference top image (a) using three markers. Each column shows evolutions from the same marker. In the second row the markers $(t=0)$ are shown, in the third and fourth rows two evolutions at $t=10 \Delta t$ and $t=20 \Delta t$, and in the fifth row the final levelings (after convergence). For the left column (b-e), the marker (b) was obtained from a 2D convolution of $f$ with a Gaussian of $\sigma=4$. For the middle column (f-i), the marker (f) was an opening by a square of $7 \times 7$ pixels and hence the corresponding leveling (i) is a reconstruction opening. For the right column $(j-m)$, the marker $(j)$ was a closing by a square of $7 \times 7$ pixels and hence the corresponding leveling $(\mathrm{m})$ is a reconstruction closing $(\Delta x=\Delta y=1, \Delta t=0.25)$. 


\section{CONCLUSIONS AND DISCUSSION}

A new morphological scale-space representation has been presented based on a general class of morphological strong filters, the levelings, with many desirable features of a scale-space. The algebraic and scale-space properties of this leveling scale-space have been analyzed using tools from mathematical morphology and illustrated with image experiments. Further, a PDE formulation has been developed for generating levelings as limits of a switched-dilation growth.

Next, we briefly discuss various issues related to levelings which include some envisioned applications, motivations for their PDE formulation, their use to improve the Gaussian scalespace, and some directions for future research.

\subsection{Some E nvisioned Applications}

In general, levelings are nonlinear filters with many interesting properties for image enhancement and simplification. This coupled with the corresponding scale-space representation developed in this paper makes them useful for numerous multiscale image analysis and vision tasks. For example, the leveling scale-space has been applied with success to reduce the bitstream of an MPEG-4 encoder, when the simplified sequence replaces the original sequence. In this case, a sliding temporal window is processed and treated as a 3D volume, with two spatial dimensions and one temporal dimension: 3D markers and 3D levelings are then used. Another important application is the simplification of the images prior to segmentation. Since the levelings enlarge flat zones, these flat zones may be used as seeds for a segmentation algorithm.

\subsection{Why U se PDE s For Levelings?}

In addition to the well-known advantages of the PDE approach (such as more insightful mathematical modeling, more connections with physics, better isotropy, better approximation of Euclidean geometry, and subpixel accuracy), during construction of levelings or reconstruction filters it is possible in some applications to need to stop the marker growth before convergence. In such cases, the isotropy of the partially grown marker offered by the PDE is an advantage. Further, there are no simple digital algorithms for constructing levelings with quasi-flat zones, whereas for the PDE approach only a simple change of the generator is needed, as we have demonstrated with the PDE producing levelings with parabolic zones.

\subsection{From $\mathrm{G}$ aussian Scale-Space to Multiscale Levelings}

Consider the hierarchical scenario of Eq. (2) to produce multiscale levelings of a reference signal $f$ based on a sequence of multiscale markers $g_{i}, i=1,2,3, \ldots$. The sequence of markers $g_{i}$ may be obtained from $f$ in any meaningful way. A particularly interesting choice we consider next is the case where the $g_{i}$ are multiscale convolutions of $f$ with Gaussians of increasing standard deviations $\sigma_{i}$. Examples of constructing multiscale levelings from Gaussian convolution markers according to Eq. (2) are shown in Fig. 6 for an image $f$. The sequence of the multiscale markers can be viewed as a scale-sampled Gaussian scalespace. As shown in the experiments, the image edges and boundaries which have been blurred and shifted by the Gaussian scale-space are better preserved across scales by the 


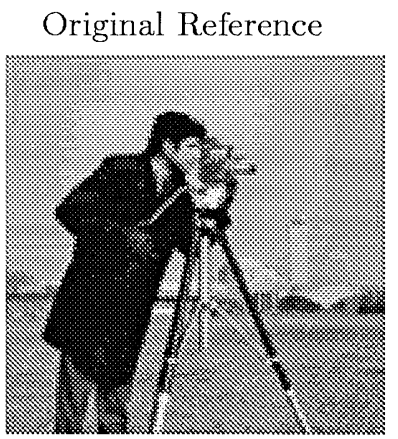

Marker 1

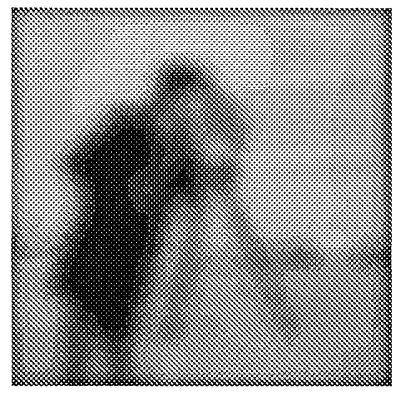

Marker 2

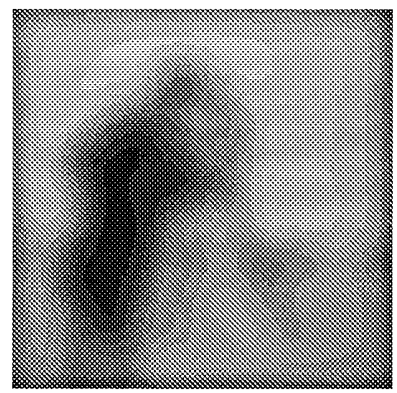

Marker 3

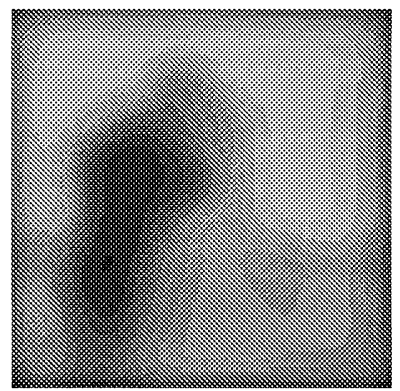

Leveling 1

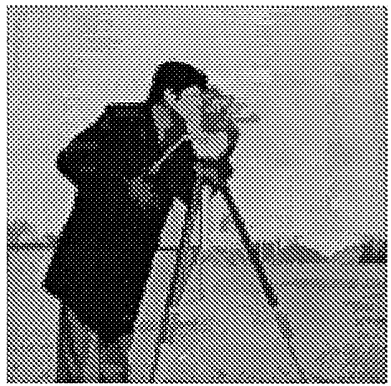

Leveling 2

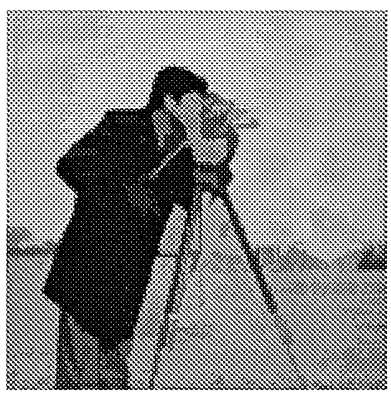

Leveling 3

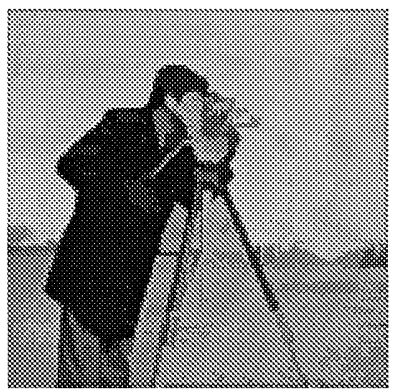

FIG. 6. Multiscale image levelings. The markers were obtained by convolving reference image with 2D Gaussians of standard deviations $\sigma=3,5,7$. (The levelings were produced by running the leveling PDE with $\Delta x=\Delta y=1, \Delta t=0.25$.) 
multiscale levelings that use the Gaussian convolutions as markers. Thus, several computer vision applications that employ the Gaussian scale-space may benefit by using the Gaussian scale-space as a first phase and the above multiscale leveling scheme as a second phase that sharpens the Gaussian convolutions toward the original image.

\subsection{Future R esearch}

Given the attractive properties of levelings and their scale-space formulations in this paper, there are many interesting directions for research on this nonlinear scale-space. Some ideas, which we plan to investigate in future papers, include the following: (1) alternative systematic approaches for selecting a sequence of markers for multiscale levelings; (2) proof of existence and uniqueness of the solution of the leveling PDE; (3) proof of convergence of the numerical algorithm implementing this PDE; (4) comparison of the complexity of the PDE-based numerical algorithm versus that of the discrete algorithm for constructing levelings based on their algebraic properties; (5) continuous-scale levelings, corresponding semigroups, and possible PDE formulation.

\section{ACKNOWLEDGMENTS}

We thank Professors J. Barrera and R. Lotufo and the State of São Paulo, Brazil, for inviting us to talk at SIBGRAPI'98 in Brazil. The ideas in this paper were formed from our collaboration during this research visit.

\section{REFERENCES}

1. L. Alvarez, F. Guichard, P. L. Lions, and J. M. Morel, Axioms and fundamental equations of image processing, Archiv. Ration. Mech. Anal. 123(3), 1993, 199-257.

2. L. Alvarez, P.-L. Lions, and J.-M. Morel, Image selective smoothing and edge detection by nonlinear diffusion, II, SIAM J. Numer. Anal. 29, 1992, 845-866.

3. A. Arehart, L. Vincent, and B. Kimia, Mathematical morphology: The Hamilton-Jacobi connection, in Proc. International Conf. Comp. Vision, 1993, pp. 215-219.

4. M. Baudin, J. Babaud, A. P. Witkin, and R. O. Duda, Uniqueness of the Gaussian kernel for scale-space filtering, IEEE Trans. Pattern Anal. Mach. Intell. 8, 1986, 26-33.

5. R. Brockett and P. Maragos, Evolution equations for continuous-scale morphological filtering, IEEE Trans. Signal Process. 42, 1994, 3377-3386. [Also in Proc. ICASSP-92, San Francisco, 1992]

6. R. A. Hummel and B. C. Gidas, Zero Crossings and the Heat Equation, Technical Report, Computer Science Division, Courant Institute of Mathematical Sciences, New York University, 1984.

7. P. T. Jackway and M. Deriche, Scale-space properties of multiscale morphological dilation-erosion, IEEE Trans. Pattern Anal. Mach. Intell. 18(1), 1996, 38-51.

8. J. J. Koenderink, The structure of images, Biol. Cybernet. 50, 1984, 363-370.

9. P. D. Lax, Hyperbolic Systems of Conservation Laws and the Mathematical Theory of Shock Waves, SIAM, Philadelphia, 1973.

10. T. Lindeberg, On scale selection for differential operators, in Proc. 8th Scandinavian Conf. Image Analysis, Norway, 1993 (B. Braathen, K. Heia, and K. A. Hogdra, Eds.), Norwegian Society for Image Processing and Pattern Recognition.

11. P. Maragos, Pattern spectrum and multiscale shape representation, IEEE Trans. Pattern Anal. Mach. Intell. 11, 1989, 701-716.

12. P. Maragos, Differential morphology and image processing, IEEE Trans. Image Process. 78, 1996, 922-937.

13. P. Maragos and F. Meyer, Nonlinear PDEs and numerical algorithms for modeling levelings and reconstruction filters, in Scale-Space Theories in Computer Vision, Lecture Notes in Computer Science, Vol. 1682, pp. 363374, Springer-Verlag, Berlin/New York, 1999. [Proc. 2nd Int'l. Conf. Scale-Space'99, Corfu, Greece, 1999] 
14. G. Matheron, Random Sets and Integral Geometry, Wiley, New York, 1975.

15. G. Matheron, Les Nivellements, Technical Report, Centre de Morphologie Mathématique, 1997.

16. F. Meyer, From connected operators to levelings, in Mathematical Morphology and Its Applications to Image and Signal Processing (H. Heijmans and J. Roerdink, Eds.), pp. 191-198, Kluwer Academic, Dordrecht/ Norwell, MA, 1998.

17. F. Meyer, The levelings, in Mathematical Morphology and Its Applications to Image and Signal Processing (H. Heijmans and J. Roerdink, Eds.), pp. 199-207, Kluwer Academic, Dordrecht/ Norwell, MA, 1998.

18. F. Meyer and P. Maragos, Morphological scale-space representation with levelings, in Scale-Space Theories in Computer Vision, Lecture Notes in Computer Science, Vol. 1682, pp. 187-198, Springer-Verlag, Berlin/New York, 1999. [Proc. 2nd Int'1. Conf. Scale-Space'99, Corfu, Greece, 1999]

19. S. Osher and L. I. Rudin, Feature-oriented image enhancement using shock filters, SIAM J. Numer. Anal. 27(4), 1990, 919-940.

20. S. Osher and J. Sethian, Fronts propagating with curvature-dependent speed: Algorithms based on HamiltonJacobi formulations, J. Comput. Phys. 79, 1988, 12-49.

21. P. Perona and J. Malik, Scale-space and edge detection using anisotropic diffusion, Proc. IEEE Computer Soc. Workshop on Computer Vision, 1987.

22. P. Perona and J. Malik, Scale-space and edge detection using anisotropic diffusion, IEEE Trans. Pattern Anal. Mach. Intell. 12, 1990, 629-639.

23. P. Salembier and J. Serra, Flat zones filtering, connected operators, and filters by reconstruction, IEEE Trans. Image Process. 4, 1995, 1153-1160.

24. G. Sapiro, R. Kimmel, D. Shaked, B. Kimia, and A. Bruckstein, Implementing continuous-scale morphology via curve evolution, Pattern Recognition 26(9), 1993, 1363-1372.

25. J. Serra, Image Analysis and Mathematical Morphology, Vol. II, Chapter on alternating sequential filters, Academic Press, San Diego, 1988.

26. J. Serra, Quelques Propriétés des Nivellements, Technical Report 30/98/MM, Centre de Morphologie Mathématique, 1998.

27. J. Serra, Set connections and discrete filtering, in Discrete Geometry for Computer Imagery, Lecture Notes in Computer Science, Vol. 1568, pp. 191-207, Springer-Verlag, Berlin/New York, 1999.

28. R. Van den Boomgaard and A. Smeulders, The morphological structure of images: The differential equations of morphological scale-space, IEEE Trans. Pattern Anal. Mach. Intell. 16, 1994, 1101-1113.

29. L. Vincent, Morphological grayscale reconstruction in image analysis: Applications and efficient algorithms, IEEE Trans. Image Process. 2(2), 1993, 176-201.

30. J. Weickert, Theoretical foundations of anisotropic diffusion in image processing, in Computing Supplement, Vol. 11, pp. 221-246, Springer-Verlag, Berlin/New York, 1996.

FERNAND MEYER received an engineering degree from the Ecole des Mines de Paris in 1975. He has worked since 1975 at the Centre de Morphologie Mathematique (CMM) of the Ecole des Mines de Paris, where he is currently a professor. His first research area was "early and automatic detection of cervical cancer on cytological smears," which was also the subject of his Ph.D. thesis. He obtained his Ph.D. in 1979. His current research fields are multiscale segmentation, morphological filtering, and processing of video sequences. His fields of application are medical imaging and multimedia applications. He holds several patents on the implementation of morphological transforms and on the construction and use of levelings.

PETROS MARAGOS received the Diploma in electrical engineering from the National Technical University of Athens in 1980, and the M.S.E.E. and Ph.D. from Georgia Tech, Atlanta, in 1982 and 1985, respectively. In 1985 he joined the faculty of the Division of Applied Sciences at Harvard University, where he worked for 8 years as a professor of electrical engineering, affiliated with the interdisciplinary Harvard Robotics Lab. He has also been a consultant to several industry research groups including Xerox's research on document image analysis. In 1993, he joined the ECE faculty of Georgia Tech. During parts of 1996-1998 he worked as a senior researcher at the Institute for Language and Speech Processing in Athens. In 1998, he joined the faculty of the National Technical University of Athens, where he is currently working as a professor of electrical and computer engineering. His current research interests include the general areas of signal processing, systems theory, control, pattern recognition, and their applications to image processing and computer vision, and computer speech processing and recognition. His professional activities in societies of signal, image, and speech processing and communications include serving 
as an editorial board member for IEEE Transactions and the VCIR Journal, chair of conferences and workshops, and member of IEEE technical committees. Dr. Maragos' research work has received several awards, including a 1987 U.S. National Science Foundation Presidential Young Investigator Award; the 1988 IEEE ASSP Paper Award for the paper "Morphological Filters"; the 1994 IEEE Signal Processing Society's Senior Award and the 1995 IEEE Baker Award for the paper "Energy Separation in Signal Modulations with application to Speech Analysis"; and the 1996 Pattern Recognition Society's Honorable Mention Award for the paper "Min-Max Classifiers." In 1995, he was elected a Fellow of IEEE. 